Molecular dynamics with Langevin equation using local harmonics and Chandrasekhar's convolution

Attila AskarRobert G. OwensHerschel A. Rabitz

Citation: The Journal of Chemical Physics 99, 5316 (1993); doi: 10.1063/1.465975

View online: http://dx.doi.org/10.1063/1.465975

View Table of Contents: http://aip.scitation.org/toc/jcp/99/7

Published by the American Institute of Physics

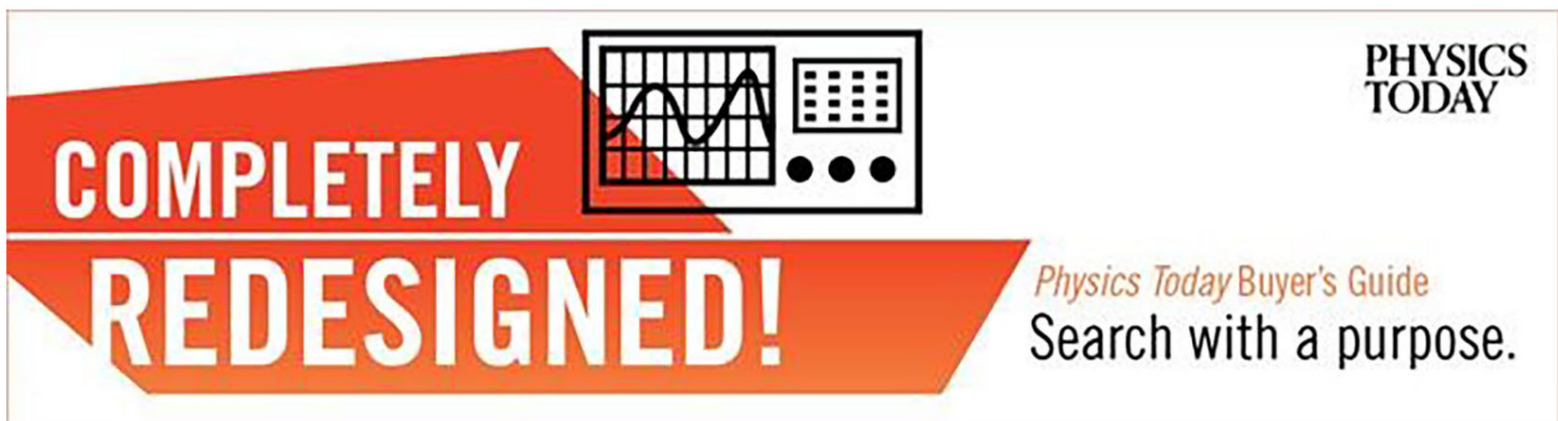




\title{
Molecular dynamics with Langevin equation using local harmonics and Chandrasekhar's convolution
}

\author{
Attila Askar \\ Koç University, Mathematics Department, Istinye, Istanbul 80860, Turkey \\ Robert G. Owens \\ Bogaziçi University, Mathematics Department, Bebek, Istanbul 80815, Turkey \\ Herschel A. Rabitz \\ Princeton University, Chemistry Department, Princeton, New Jersey 08544
}

(Received 18 March 1993; accepted 17 July 1993)

\begin{abstract}
A numerical method for studying molecular systems subject to a random force field leading to a Gaussian velocity distribution and described by the Langevin equation is presented. Two basic elements constitute the formulation: local harmonic modes and Chandrasekhar's formula for the distribution function for a convolution involving a random function. First, by linearizing the governing Langevin equations locally and employing an orthogonal change of coordinates, an explicit solution for the displacement and velocity is constructed. Second, Chandrasekhar's formula is employed in deriving the probability distribution function of the displacements and the velocities coming from the random forces. The local mode analysis is essential for the use of the Chandrasekhar's formula, since we need the formal solution as a convolution of the random forces and the local Green's function. For an illustration of the method in a significant case representative of real problems, we study a one dimensional idealization of a long chain molecule possessing internal energy barriers and subjected to an applied tension. The results are compared with the predictions of a conventional approximate method where a finite number of random realizations are generated in each time step. This truncation constitutes an approximation to obtain the desired Gaussian probability distribution function for the velocities which is reached in the limit of an infinity of random realizations. The calculations show that the conventional approximations may be acceptable only for short times, small temperatures, and average values over very long times. In particular, these approximations fail to give accurate results for transient phenomena, show slow convergence with the increase in the number of random realizations, and predict large values for the variance even in the steady regime. The new proposed method on the other hand, (i) incorporates the mathematically and conceptually correct limit for the distribution function, (ii) is quite stable with respect to increases in the value of the time increment as well as in terms of fluctuations characterized by the variance, (iii) leads to considerable savings in computer time over the approximate method, and (iv) has the proper description during the transient regime, which is usually the most interesting phase of dynamical processes.
\end{abstract}

\section{INTRODUCTION}

The numerical simulation of chain molecules in a solution has been the focus of much previous research. ${ }^{1,2}$ In studying Brownian motion, we are concerned with the random oscillations induced in particles immersed in a fluid. Under normal conditions, a particle will suffer about $10^{13}-10^{15}$ collisions per second so that following the particle path is impossible. Simulations therefore are concerned with statistical information about the positions and velocities of the atoms for periods which are long with respect to a time step. Two elements in the numerical technique are important. First, the manner in which the Brownian motion effect on the atoms is computed. Second, the way that the differential equation governing the molecular motion is solved numerically.

In this paper we present a numerical method for studying molecular systems subject to a random force field leading to a Gaussian velocity distribution and described by the Langevin equation. Methods of conducting "Brownian dy" namics" simulations have been developed. ${ }^{1-23}$ These can be grouped into two general lines of thought. The most straightforward idea is to consider random force field realizations acting on the system during a time interval $\Delta t$ and to integrate the equations as if the generated force field is deterministic in $\Delta t$. In practice, this leads to generating random force fields taken as constants over time subintervals of length $\delta t$ within the considered $\Delta t$. For a random force field having fixed amplitude of positive or negative sign with equal probability on each $\delta t$, the corresponding velocity field has a Gaussian distribution. This happens, however, only in the limit of an infinity of random realizations within each time step, i.e., for $\lim \Delta t / \delta t \rightarrow$ infinity. ${ }^{3-5,24}$ This consideration identifies a key approximation, since actual calculations are limited by $\Delta t / \delta t=M$ being finite. The consequences of this approximation as well as the rate of convergence with increasing $M$ need to be investigated. Of course, in this approach, the system of equations is deterministic for each random realization and can 
be integrated directly using, for instance, a Verlet, RungeKutta, or Gear algorithm. The second line of thought ${ }^{1,11-13,17,18}$ considers the equations for the momentum with the damping term present and hence deals with first order equations. In this organization of the dynamical equations, the interaction forces are considered explicitly on the right-hand side as a function of time by substituting in the values of the position coordinates from the preceding time step. The equations are discretized basically as polynomials in time and the contributions of the random forces are calculated by convolutions. Due to writing the equations as first order in the momentum, the kernels are exponential functions. The distribution function of these integrals is determined by using Chandrasekhar's formula. ${ }^{24}$ In this type of formulation the interaction force field is considered as a constant or a linear function in time during the discrete time interval $\Delta t$. The discretization of the equations is done by a Verlet or Runge-Kutta formula for the first order derivatives.

A comment here is in order in relating the small but finite time interval $\Delta t$ to Chandrasekhar's formulation. The random and uncorrelated field will ideally lead to a Gaussian velocity distribution for any interval of time, however small, including those of the order of the discretized $\Delta t$ for the numerical integration. Chandrasekhar considered a single interval and $\Delta t$ in his notation is our $\delta t$. Similarly, our $\Delta t$ corresponds to the finite time interval in this latter work. $\Delta t$ is an interval sufficiently small where the systematic forces, i.e., the interaction forces, are considered as constant. This interval is nevertheless still large for the random forces to be taken as constant. It is precisely why many realizations are needed for accounting for the rapid changes of the random forces within this time interval $\Delta t$. The concept of the length of the interval is a relative one and this is precisely defined by the ratio $\Delta t / \delta t$ which is required to go to the infinity limit. A similar discussion can be found in Refs. 3 to 6 . This issue is commonly addressed by stating that although the force field does not lead to a Gaussian velocity distribution in each $\Delta t$, the necessary distribution is achieved in a practical sense for the averages over the whole length of the process involving typically an interval of several thousand $\Delta t$ 's. One of the important considerations in this paper is to clarify this conjecture, and in particular to understand what happens in the transient regime in time as well as to establish how long it takes to reach the steady state.

The present method bears similarities in spirit to the second line of thought ${ }^{1,11-13,17,18}$ where the distribution function for integrals involving the random forces is also calculated by using Chandrasekhar's formula. However, the equations in the present case consider the interaction forces as dominant to the extent that the kernel of the convolutions with the random forces are damped oscillator functions. In this sense, the schemes proposed in these latter references are more appropriate for liquids where the damping is dominant, whereas the present method is better suited to solids where the interaction forces are dominant. In addition, in the proposed scheme, the dynamical variables vary trigonometrically in time as opposed to the poly- nomials as in the Verlet or Runge-Kutta based algorithms. As a result, large time steps are acceptable with the present scheme. Appendix A compares the solution of the harmonic oscillator problem by the algorithms of Ermak ${ }^{1,11-13}$ and Allen ${ }^{1,17,18}$ with the present one in order to clarify the differences and similarities of the two approaches. However, in this article, there is no comparisons with numerical results based on these latter algorithms. Work along these lines is the subject of a future investigation. For an illustration of the proposed method in a significant case representative of real problems, a one dimensional idealization of a long chain molecule possessing internal energy barriers and subjected to an applied tension is studied. This model was introduced by Weiner and Pear ${ }^{3}$ and is described in Appendix B. The numerical comparisons are carried out between the proposed scheme and the more conventional methods along the first line of thought.

In Sec. II, we describe the Langevin equation governing the behavior over time of the dynamical system starting from known initial conditions and subject to a random force field which is uncorrelated between different points in the chain and has a Gaussian momentum distribution. In Sec. III we show two ways in which the effect of the random force acting on the system may be accounted for. The first one is a traditional integration based on generating several realizations of a random force field during each time interval, thereby approximating the Gaussian probability distribution for the impulsive forces as best as possible. Indeed, the correct momentum distribution is reached only in the limit of an infinity of random realizations. In our numerical evaluations, we considered 1,10 , and 20 realizations in each time interval $\Delta t$. In the second scheme, which is the method proposed in the present article, we introduce an exact and efficient way of computing the output due to the random forces by using Chandrasekhar's formula. This scheme hence uses the exact result for the probability distribution for convolutions involving the Green's function and the random force field. Finally, in Sec. IV, the numerical results are presented with both the approximate method with 1,10 , and 20 random realizations in each interval along with the proposed method. Some general observations are reported in Sec. V.

\section{GOVERNING EQUATIONS}

The equation and the initial conditions governing the dynamics of the molecular system are taken in nondimensional units to be

$$
\begin{aligned}
& \ddot{\mathbf{x}}=-\mathbf{f}(\mathbf{x})-2 \eta \dot{\mathbf{x}}+\mathbf{R}(\mathbf{t}), \\
& \mathbf{x}(0)=\mathbf{x}_{\mathbf{0}} \quad \dot{\mathbf{x}}(0)=\dot{\mathbf{x}}_{\mathbf{0}} .
\end{aligned}
$$

Above, $\mathbf{x}$ denotes the position vector of the assembly of atoms and for a system having $n$ atoms is a vector in $3 n$ dimensions; $f_{i}=\partial V / \partial \mathbf{x}_{i}$ is the $i$ th component of the force acting on the particles as derived from the interatomic potential $V=V(\mathbf{x}) ; \eta$ is a damping factor and the vector $\mathbf{R}=\mathbf{R}(t)$ is the random external force acting on the system and satisfying the statistical relations ${ }^{5}$ 


$$
\begin{aligned}
& \left\langle R_{i}(t)\right\rangle=0, \\
& \left\langle R_{i}(t) R_{j}\left(t^{\prime}\right)\right\rangle=4 \eta T \delta\left(t-t^{\prime}\right) \delta_{i j},
\end{aligned}
$$

where $T$ is the nondimensional temperature with the Planck's constant absent, the $R_{i}$ are the components of $\mathbf{R}$, and \langle\rangle denotes an average taken over the atoms. $\eta$ and $\mathbf{R}$ model temperature effects on the molecule in the sense of the Langevin equation for Brownian motion. $\mathbf{x}_{0}$ and $\dot{\mathbf{x}}_{0}$ denote, respectively, the initial conditions on the position vector and velocity. Consider the coordinate transformation

$$
\mathbf{x}=\mathbf{x}_{0}+\mathbf{u},
$$

where $\mathbf{u}$ is the displacement about $\mathbf{x}_{0}$. With $|\mathbf{u}|<\left|\mathbf{x}_{0}\right|$, a linearization of Eq. (2.1) around the initial state using Taylor's theorem yields

$$
\begin{aligned}
& \ddot{\mathbf{u}}=-\mathbf{H} \cdot \mathbf{u}-2 \eta \dot{\mathbf{u}}-\mathbf{f}^{0}+\mathbf{R}(t), \\
& \mathbf{u}(0)=\mathbf{0}, \quad \dot{\mathbf{u}}(0)=\dot{\mathbf{x}}_{0},
\end{aligned}
$$

where $f^{0}=f\left(x_{0}\right)$ is a constant vector reflecting the nonlinearity and $\mathbf{H}$ is the Hessian or the Jacobian of the interaction potential $V$. That is

$$
\mathbf{f}^{0}=\mathbf{f}\left(\mathbf{x}_{0}\right)=\operatorname{grad} V\left|\mathbf{x}_{0} \quad \mathbf{H}=\operatorname{grad} \operatorname{grad} V\right| \mathbf{x}_{0} .
$$

Towards the solution of the linearized system of equations in Eq. (2.4), consider the orthogonal coordinate transformation

$$
\mathbf{u}=\mathbf{Q} \cdot \mathbf{z},
$$

where $\mathbf{Q}$ is the orthogonal matrix of normalized eigenvectors of $\mathbf{H}$. The substitution of Eq. (2.6) into Eq. (2.4) and the premultiplication throughout by $\mathbf{Q}^{T}$ yields

$$
\ddot{\mathbf{z}}=-\mathbf{Q}^{T} \cdot \mathbf{H} \cdot \mathbf{Q} \cdot \mathbf{z}-2 \eta \dot{\mathbf{z}}-\mathbf{Q}^{T} \cdot \mathbf{f}^{0}+\mathbf{Q}^{T} \cdot \mathbf{R} .
$$

In component form, the above equation reads

$$
\ddot{z}_{k}=-\lambda_{k} z_{k}-2 \eta \dot{z}_{k}-\sum_{m=1}^{n} Q_{k m}^{T} f_{m}^{0}+\sum_{m=1}^{n} Q_{k m}^{T} R_{m},
$$

where $\lambda_{k}$ is the $k$ th eigenvalue of the matrix $\mathbf{H}$. The solution of Eq. (2.8) may be written collectively in matrix form as

$$
\begin{aligned}
\mathbf{z}= & (\mathbf{C} \cdot \mathbf{a}+\mathbf{S} \cdot \mathbf{b}) e^{-\eta t}+\mathbf{L} \cdot \mathbf{Q}^{T} \cdot \mathbf{f}^{0} \\
& +\int_{0}^{t} \mathbf{G}\left(t-t^{\prime}\right) \cdot \mathbf{Q}^{T} \cdot \mathbf{R}\left(t^{\prime}\right) d t^{\prime},
\end{aligned}
$$

where the elements of $\mathbf{a}$ and $\mathbf{b}$ are (as yet) unknown integration constants. Depending on the value of $\lambda_{k}-\eta^{2}$ as positive, zero, and negative the elements of the diagonal matrices $\mathbf{C}, \mathbf{S}$, and $\mathbf{G}$ are given, respectively, by one of the values in parentheses in the following expressions:

$$
\begin{aligned}
\mathbf{C}_{k l}= & \left\{\cos \omega_{k} t, 1, \cosh \omega_{k} t\right\} \delta_{k l}, \\
\mathbf{S}_{k l}= & \left\{\sin \omega_{k} t / \omega_{k}, t, \sinh \omega_{k} t / \omega_{k}\right\} \delta_{k l}, \\
\mathbf{G}_{k l}= & e^{-\eta\left(t-t^{\prime}\right)}\left\{\sin \omega_{k}\left(t-t^{\prime}\right) / \omega_{k}, t-t^{\prime},\right. \\
& \left.\sinh \omega_{k}\left(t-t^{\prime}\right) / \omega_{k}\right\} \delta_{k l},
\end{aligned}
$$

where

$$
\omega_{k}^{2}=\left|\lambda_{k}-\eta^{2}\right| .
$$

The elements $L_{k l}$ of the diagonal matrix $\mathbf{L}$ corresponding to the particular solution for the constant vector $\mathrm{f}^{0}$ is given by

$$
\begin{array}{r}
-1 / \lambda_{k}+\left(e^{-\eta t} / \omega_{k} \lambda_{k}\right)(\eta \sin \omega t+\omega \cos \omega t) \delta_{k l} \\
\text { if } \lambda_{k}-\eta^{2}>0, \\
-1 / \lambda_{k}+\left(e^{-\eta t} / \omega_{k} \lambda_{k}\right)(\eta \sinh \omega t+\omega \cosh \omega t) \delta_{k l} \\
\text { if } \lambda_{k}-\eta^{2}<0,
\end{array}
$$

$-\left(1 / \eta^{2}\right)+\left(e^{-\eta t} / \eta^{2}\right)(1+\eta t) \delta_{k l}$

$$
\text { if } \lambda_{k}=\eta^{2}
$$

$-(t / 2 \eta)+\left(e^{\left.-\eta t / 2 \eta^{2}\right)(\sinh \eta t) \delta_{k l}}\right.$

$$
\text { if } \lambda_{k}=0 \text {. }
$$

The solution for $\mathbf{u}$ and subsequently $\mathbf{x}$ is obtained by the inverse transformation of $\mathbf{z}$ and in vector form may be written as

$$
\begin{aligned}
\mathbf{x}= & \mathbf{x}_{0}+\mathbf{u}^{0}+e^{-\eta t}(\mathscr{C} \cdot \mathbf{A}+\mathscr{S} \cdot \mathbf{B}) \\
& +\int_{0}^{t} \mathscr{G}\left(t-t^{\prime}\right) \cdot \mathbf{R}\left(t^{\prime}\right) d t^{\prime}, \\
\dot{\mathbf{x}}= & \dot{\mathbf{u}}^{\mathbf{0}}+e^{-\eta t}[(\dot{\mathscr{C}} \cdot \mathbf{A}+\mathscr{S} \cdot \mathbf{B})-\eta(\mathscr{C} \cdot \mathbf{A}+\mathscr{S} \cdot \mathbf{B})] \\
& +\int_{0}^{t} \dot{\mathscr{G}}\left(t-t^{\prime}\right) \cdot \mathbf{R}\left(t^{\prime}\right) d t^{\prime},
\end{aligned}
$$

where

$$
\begin{aligned}
& \mathbf{u}^{0}=\mathbf{L} \cdot \mathbf{f}^{0}, \quad \mathscr{G}\left(t-t^{\prime}\right)=\mathbf{Q} \cdot \mathbf{G}\left(t-t^{\prime}\right) \cdot \mathbf{Q}^{T}, \\
& \mathscr{C}=\mathbf{Q} \cdot \mathbf{C} \cdot \mathbf{Q}^{T}, \quad \mathscr{S}=\mathbf{Q} \cdot \mathbf{S} \cdot \mathbf{Q}^{T}, \quad \mathscr{L}=\mathbf{Q} \cdot \mathbf{L} \cdot \mathbf{Q}^{T},
\end{aligned}
$$

$\mathbf{A}$ and $\mathbf{B}$ are related to $\mathbf{a}$ and $\mathbf{b}$ in Eq. (2.9) and are determined from the initial conditions as

$$
\mathbf{A}=\mathbf{Q} \cdot \mathbf{a}=\mathbf{0}, \quad \mathbf{B}=\mathbf{Q} \cdot \mathbf{b}=\dot{\mathbf{x}}_{\mathbf{0}} .
$$

Due to the diagonal nature of the matrices $\mathbf{C}, \mathbf{S}, \mathbf{L}$, and $\mathbf{G}$, the component representations of the matrices $C, S, L, G$ are of the forms

$$
\mathscr{C}_{k l}=\sum_{m=1}^{n} Q_{k m} Q_{l m} \mathrm{C}_{m m},
$$

along with similar expressions for the other matrices.

The formulas above allow us to propagate the solution at time $t=0$ to time $\Delta t$ selected sufficiently small to validate the local linearization. By repeating the process in subsequent time intervals, it is possible to march in time. Two questions, those of accuracy and stability arise. At this point we do not have a rigorous theoretical study of these matters. In our calculations we take repeated calculations with two different time steps $\Delta t$ in order to ensure the accuracy and stability. In the next sections we study the particular case where the external force is random. 


\section{QUADRATURE WITH THE RANDOM FORCE}

In this section, two methods for the evaluation of the convolutions with the random vector $\mathbf{R}$ in Eq. (2.13) are presented. The purpose here is to clarify the importance of the finiteness of the number of realizations of the random and uncorrelated forces in each time interval $\Delta t$. In the first set of calculations only a finite number of realizations is possible, while the second scheme corresponds to the limit of infinity of random realizations. In principle, the original system defined in Eq. (2.2) can be integrated directly using for instance a Verlet, Runge-Kutta, or Gear algorithm. However, in clarifying the effect of the finite number of random realizations in each time interval, the integration method is kept the same, i.e., the method presented here based on a local linearization of the interaction forces and the use of the eigenvalues and eigenvectors of the associated Jacobian. The only difference between the two schemes is in the way the random term is handled. In the first scheme, the convolution of the Green's function with the random forces in Eq. (2.13) is evaluated through a quadrature by generating the random forces at $M$ equally spaced points within the time intervals $\Delta t$. The second scheme finds the exact probability distribution of the convolution with the random force using the Chandrasekhar formula and then generates the contribution of this term to the displacements of the particles in the molecular system using a random number generator.

\section{A. Direct evaluation of the convolutions with the random force}

During each interval $[0, \Delta t]$, the random force $\mathbf{R}$ undergoes many fluctuations so that the integral expression in Eq. (2.13) over this interval is evaluated as the sum of $M$ integrals over subintervals $[k \delta t,(k+1) \delta t]$ with $\delta t=\Delta t / M$ and $k=0,1,2, \ldots, M-1$. The random force components $R_{j}$ are made to assume a constant value

$$
R_{j}=2 \sqrt{\frac{\eta^{\dagger} T}{\delta t}}(-1)^{\theta}
$$

over each subinterval of length $\delta t$, where $\theta$ is an even or odd integer with equal probability and $T$ is the temperature. This choice for the components $R_{j}$ of $\mathbf{R}$ ensures (see Appendix $C$ ) that as $\delta t / \Delta t \rightarrow 0$, i.e., in the limit of an infinity of random samplings in the time interval $\Delta t$, the integral

$$
B_{j}(\Delta t)=\int_{0}^{\Delta t} R_{j}\left(t^{\prime}\right) d t^{\prime}
$$

has the Gaussian probability distribution with zero mean and variance $\sigma_{j}^{2}$ given by

$$
\sigma_{j}^{2}=4 \eta T \Delta t
$$

This is the distribution of the impulse derived from the random force $\mathbf{R}$ in Eq. (2.2) ${ }^{24}$ The obvious drawback with this method is that it requires many "inner" time steps of length $\delta t$ for each outer time step of length $\Delta t$ for the integral $B_{j}$ to assume the correct statistical properties.

\section{B. Evaluation of the convolutions with the random force by Chandrasekhar's method}

The integral in Eq. (2.13) involves the random vector R. Let us consider

$$
I_{l}=\int_{0}^{\Delta t} \psi\left(\Delta t-t^{\prime}\right) R_{l}\left(t^{\prime}\right) d t^{\prime}
$$

where $\psi$ is a deterministic function. This integral is a generalization of that in Eq. (3.2) and is a random variable having a Gaussian probability distribution with zero mean and with the variance given by ${ }^{24}$

$$
\Sigma^{2}=4 \eta T \int_{0}^{\Delta t} \psi^{2}\left(\Delta t-t^{\prime}\right) d t^{\prime}
$$

For the specific integral in Eq. (2.12), let us break the expression into component form by defining

$$
\begin{aligned}
& P_{k l}=\int_{0}^{\Delta t} G_{k k}\left(\Delta t-t^{\prime}\right) R_{l}\left(t^{\prime}\right) d t^{\prime}, \quad(\text { no sum on } k) \\
& z_{k}=\sum_{l=1}^{n} Q_{l k} P_{k l} \\
& x_{j}=\sum_{k=1}^{n} Q_{j k} z_{k} .
\end{aligned}
$$

In view of the previous discussion, all of the random variables derived from $R_{l}$ have zero mean and have a Gaussian distribution function. In particular, the variance for $P_{k l}$, following Eq. (3.5) is

$$
\operatorname{Var}\left(P_{k l}\right)=\Sigma_{k}^{2}=4 \eta T \int_{0}^{\Delta t} G_{k k}^{2}\left(\Delta t-t^{\prime}\right) d t^{\prime} .
$$

It may be shown (see Appendix C) that by replacing the components of $\mathbf{R}$ by those in Eq. (3.1) that the same variance $\Sigma_{k}^{2}$ is given in the limit as $\delta t / \Delta t \rightarrow 0$. It is important to note that although the random variable $P_{k l}$ carries both of the indices $k$ and $l$, its variance carries only the index $k$, since the variance of the integral $R_{l}$ as in Eq. (3.3) is independent of the index $l$. This property merely follows from the random external forces being uncorrelated with the same distribution and proves to be very useful for the computations. Indeed, with the second equation in Eq. (3.6), the variance of $z_{k}$ becomes

$$
\operatorname{Var}\left(z_{k}\right)=\sum_{l=1}^{n} Q_{l k}^{2} \Sigma_{k}^{2}=\Sigma_{k}^{2}
$$

This last result follows from $\mathbf{Q}$ being an orthonormal matrix, i.e., an orthogonal matrix with its columns normalized. Finally, with the variance of $z_{k}$ as above, from the third equation in Eq. (3.6), it follows that

$$
\operatorname{Var}\left(x_{j}\right)=\sum_{k=1}^{n} Q_{j k}^{2} \Sigma_{k}^{2}
$$

For each time step of length $\Delta t$ and for each component $x_{j}$ a random variable with zero mean and variance as above is generated using a routine from Numerical Recipes. ${ }^{25}$ 


\section{NUMERICAL APPLICATIONS WITH A POLYMER MODEL}

The scheme is applied to a model of a polymer chain with a quartic double well interaction potential between nearest neighbors. The polymer model is due to Weiner and Pear ${ }^{3}$ and for keeping the continuity of the mathematics for the reader not interested in the details of the polymer, the model is presented in Appendix B. Due to the nearest neighbor interactions, the Hessian matrix of the linearized form is tridiagonal. Although, of course, this is a simplifying feature in the calculations, the scheme is by no means restricted to this special case. For $V$ representing the pair interaction potential, the dot and the prime, respectively, denoting the derivatives with respect to time and coordinates, the governing dynamical equations are of the form

$$
\begin{aligned}
\ddot{x}_{j}= & -V^{\prime}\left(x_{j}-x_{j-1}\right)+V^{\prime}\left(x_{j+1}-x_{j}\right)-2 \eta \dot{x}_{j}+R_{j}(t) \\
& j=1, \ldots, n-1, \\
\ddot{x}_{n}= & \sigma-V^{\prime}\left(x_{n}-x_{n-1}\right)-2 \eta \dot{x}_{n}+R_{n}(t) \\
x_{0}= & 0 .
\end{aligned}
$$

Consequently, in the notation of Eqs. (2.1) and (2.4), we have

$$
\begin{aligned}
& f_{j}=V^{\prime}\left(x_{j}-x_{j-1}\right)-V^{\prime}\left(x_{j+1}-x_{j}\right), \quad j=1, \ldots, n-1, \\
& f_{n}=-\sigma+V^{\prime}\left(x_{n}-x_{n-1}\right), \\
& H_{j j-1}=-V^{\prime \prime}\left(x_{j}-x_{j-1}\right), \quad j=2, \ldots, n, \\
& H_{j j}=V^{\prime \prime}\left(x_{j}-x_{j-1}\right)+V^{\prime \prime}\left(x_{j+1}-x_{j}\right), \quad j=1, \ldots, n, \\
& H_{j j+1}=-V^{\prime \prime}\left(x_{j+1}-x_{j}\right), \quad j=1, \ldots, n-1,
\end{aligned}
$$

where $V(x)=\gamma\left(1-x^{2}\right)^{2}$ in Eq. (4.1) along with a nonzero damping $\eta$ and a random force of the type in Sec. III. Equation (4.2) shows that a stress $\sigma$ is applied to the end point $x_{n}$.

For the numerical calculations, the polymer chain had 15 atoms and $\Delta t, \sigma, \eta$, and $\gamma$ are fixed at $0.1,0.1,0.1$, and 0.5 , respectively. Three sets of calculations distinguished by the ambient temperature field are performed using this one dimensional polymer chain model. The calculations for each set are based on both the direct integration method of Sec. III A and the method using Chandrasekhar's formula in Sec. III B. The aim of the computations performed is to ascertain the ability of the approximate method to reproduce the results generated by the proposed scheme using Chandrasekhar's formula. For all cases, the calculations with the approximate method are conducted by choosing one, ten, and 20 inner steps. It should be noticed that the Chandrasekhar scheme corresponds to the limit of infinity of inner steps, which thereby provides the exact Gaussian distribution for the momenta.

The temperature and initial conditions distinguishing the three sets of calculations are as follows:

Case 1: Step change in the temperature. These calculations are similar to earlier calculations with the model by Weiner and Pear. ${ }^{3}$ The initial temperature of 0.0375 is

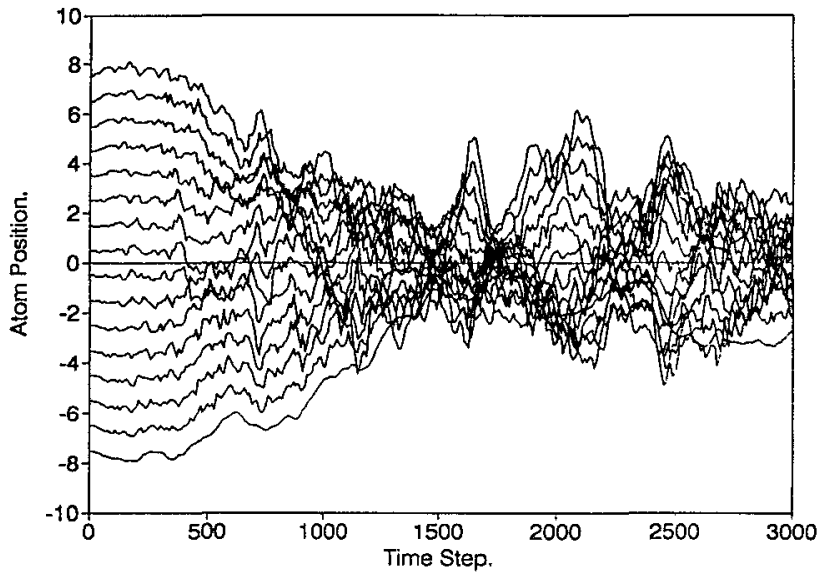

FIG. 1. A sample plot of the atomic trajectories relative to the center of mass in Case 2, calculated using Chadrasekhar's integration formula. The common parameters used in the calculations are $\Delta t=0.1, \eta=0.1, \sigma=0.1$, $\gamma=0.5$.

changed in a stepwise manner to the value of 0.15 after 1000 time steps of size $\Delta t$ and then fixed at the new temperature for 7000 time steps. The final time is judged as being sufficient for reaching the equilibrium length at this temperature. This is important in order to compare the lengths predicted by the different approaches in a meaningful way. The initial configuration is taken to be a completely stretched chain and the molecule is started from rest.

Case 2: Linear temperature increase followed by a plateau. The temperature $T$ is raised at a rate of $\dot{T}=0.0002$ per time step up until 1000 time steps and then fixed at its value of $T=0.2$ for the subsequent 7000 time steps. This loading history is chosen so as to introduce fewer transients due to the smoother change in temperature as compared with the previous case.

Case 3: The temperature $T$ is raised at the same rate of $\dot{T}=0.0002$ as in the previous calculations for the whole duration of the process up until 8000 time steps. Hence, the final value of the temperature in this case is 1.6 , i.e., an order of magnitude larger than in the previous two loading cases.

Figure 1 shows the particle trajectories for Case 2 as calculated by Chandrasekhar's formula. This figure is representative of what happens in all of the three cases studied. The differences between the results of the various types of loading histories are in the details and the complete set is not presented for economy in the presentation. Nevertheless, the quantitative differences between the various modes of calculations are significant. Similarly, Figs. 2(a), 2(b), and 2(c) show the time averaged value of the chain length as $(1 / t) \int_{0}^{t}\left[X_{n}\left(t^{\prime}\right)-X_{0}\left(t^{\prime}\right)\right] d t^{\prime}$ for the three cases. Each set of figures shows the results for $M=1,10,20$ inner loops as well as the result of the present scheme. Tables $I(a)$ and $I(b)$ summarize the results of the three cases studied in reference to the average lengths and the standard deviations in the average lengths corresponding to 1 , 10, 20 inner steps and the Chandrasekhar formula 
[see Eq. (3.9)]. The clear trend is the shortening of the molecule length from its initial value of 15 and this is in keeping with the predictions of Weiner and Pear. ${ }^{3}$ Again, in accord with physical arguments, the molecule shortens more with increasing temperature. In Table I(a), the long term equilibrium lengths are computed by averaging the value of $x_{n}-x_{0}$ of the chain length over the entire 8000 time

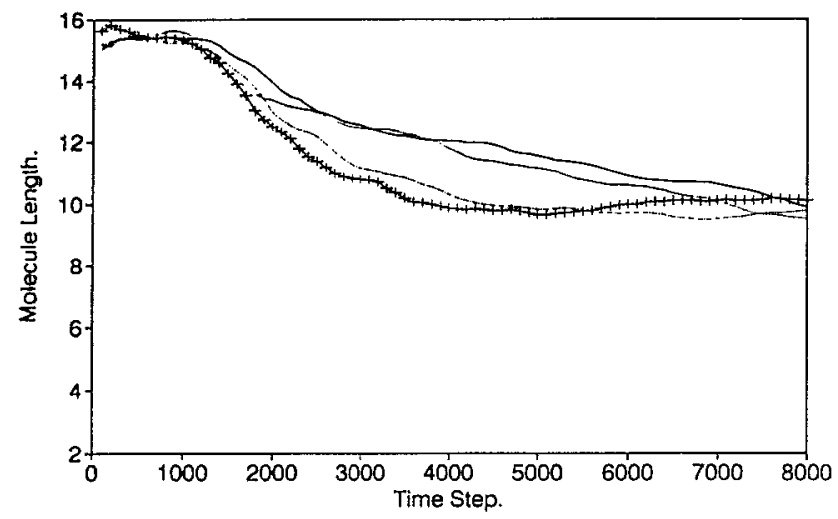

(a)

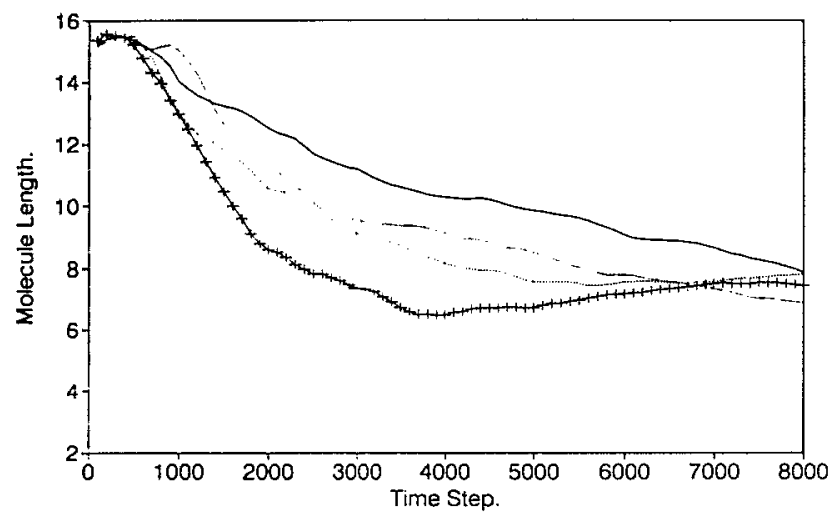

(b)

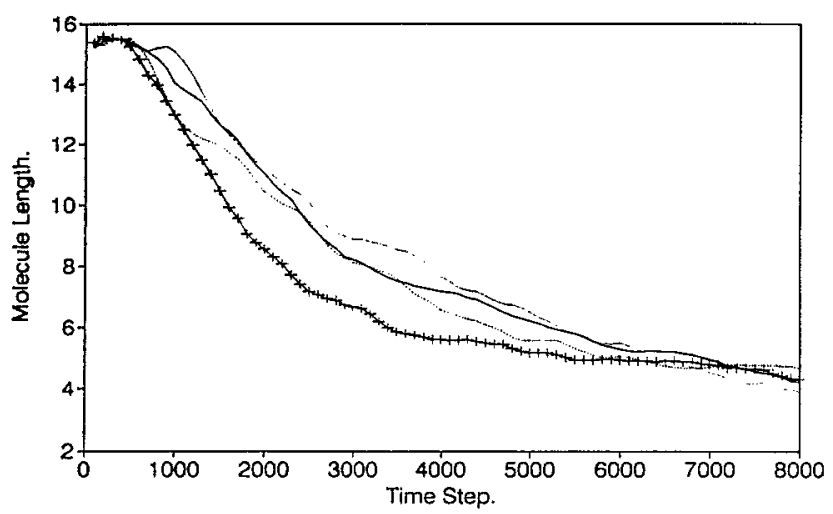

(c)

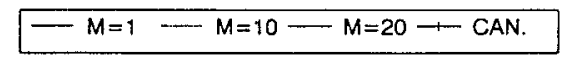

FIG. 2. (a) Case 1. Average length $x_{n}-x_{0}$ computed over time step intervals of $[0,99],[0,199], \ldots,[0,7999]$ and plotted at the end point of each interval. (b) Case 2. As above. (c) Case 3. As above.
TABLE I. (a) Chain length $x_{n}-x_{0}$ averaged over the duration of the

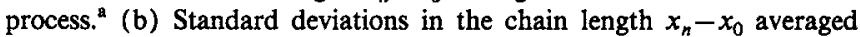
over the duration of the process.

\begin{tabular}{|c|c|c|c|c|c|c|c|c|}
\hline (a) & Case 1 & $\begin{array}{l}\text { \%age } \\
\text { error }\end{array}$ & Case 2 & $\begin{array}{l}\text { \%age } \\
\text { error }\end{array}$ & Case 3 & $\begin{array}{l}\text { \%age } \\
\text { error }\end{array}$ & \multicolumn{2}{|c|}{$\begin{array}{c}\text { CPU time per } \\
\text { iteration }(s)\end{array}$} \\
\hline$M=1$ & 9.50 & 6.2 & 6.90 & 7.6 & 3.94 & 9.0 & & \\
\hline$M=10$ & 9.80 & 3.3 & 7.82 & 4.7 & 4.70 & 8.5 & & \\
\hline$M=20$ & 9.92 & 2.1 & 7.90 & 5.8 & 4.24 & 2.1 & & \\
\hline Chand. & 10.13 & & 7.47 & & 4.33 & & & \\
\hline (b) & Case & & $\begin{array}{l}\text { \%age } \\
\text { error }\end{array}$ & Case & & $\begin{array}{l}\text { \%oage } \\
\text { error }\end{array}$ & Case 3 & $\begin{array}{l}\text { \%age } \\
\text { error }\end{array}$ \\
\hline$M=1$ & 3.35 & & 12.7 & 3.73 & & 9.6 & 5.71 & 35.2 \\
\hline$M=10$ & 3.02 & & 1.5 & 3.08 & & -9.5 & 4.66 & 10.1 \\
\hline$M=20$ & 3.22 & & 8.4 & 3.65 & & -7.2 & 4.90 & 15.9 \\
\hline Chand. & 2.97 & & & 3.40 & & & 4.23 & \\
\hline
\end{tabular}

${ }^{3}$ Three cases, with different temperature loading. Results are presented for the approximate methods and the proposed method using Chandrasekhar's formula. The common parameters used in the calculations are $\eta=0.1, \sigma=0.1, \gamma=0.1$, and $\Delta t=0.1$. The initial length of the molecule in all cases is 15 . All calculations are carried for 8000 time steps. The loading in Case 1 consists of a step change in the temperature from the initial value of 0.0375 , to a value of 0.15 after 1000 time steps. The loading in Case 2 is a linear increase in temperature at a rate of 0.0002 per time step up until 1000 time steps, which is then followed by a plateau at its value of $T=0.2$. Finally, for Case 3 , the temperature is raised at the same rate as in Case 2 for the whole duration of the process, thereby reaching the value of 1.6 .

steps for all cases. The standard deviations are computed likewise. These results show that the long term equilibrium lengths predicted by the approximate and Chandrasekhar approaches converge to a common value. The convergence to the limit value is found to be rather slow, nonuniform and even 20 realizations in each interval did not yield satisfactory results. In fact, the results for the one inner loop case show strong fluctuations. The standard deviations in Table $I(b)$ are seen to be rather erratic, in particular for the calculations with a single inner loop. Again, this indi-

TABLE II. (a) Chain length $x_{n}-x_{0}$ averaged over the duration of the process. ${ }^{\mathrm{a}}$ (b) Standard deviations in the chain length $x_{n}-x_{0}$ averaged over the duration of the process.

\begin{tabular}{lccc}
\hline \hline (a) & $\begin{array}{c}\text { Case 2 } \\
\Delta t=0.1\end{array}$ & $\begin{array}{c}\text { Case 2 } \\
\Delta t=0.5\end{array}$ & $\begin{array}{c}\text { \%age } \\
\text { error }\end{array}$ \\
\hline$M=1$ & 6.90 & 11.00 & 59.4 \\
$M=10$ & 7.82 & 11.09 & 41.8 \\
$M=20$ & 7.90 & 11.39 & 44.2 \\
Chand. & 7.47 & 7.42 & 0.7 \\
& & & \\
(b) & Case 2 & Case 2 & \%age \\
\hline$M=1$ & $\Delta t=0.1$ & $\Delta t=0.5$ & error \\
$M=10$ & 3.73 & 3.24 & -4.8 \\
$M=20$ & 3.08 & 2.83 & -16.8 \\
Chand. & 3.65 & 2.55 & -25.0 \\
\hline \hline
\end{tabular}

"Convergence study of the various modes of calculation for the second numerical experiment with $\Delta t=0.1$ and 0.5 . The total duration of the process is again the same as for Table 1 . The other parameters used in the calculations are the same as those in Table $I$. The percent error is defined for the values of $\Delta t=0.5$, relative to those for $\Delta t=0.1$. 
cates that the approximate calculations converge rather slowly and display significant errors. Table I(a) also shows the CPU time per time step on an IBM compatible 386-33 $\mathrm{MHz}$ workstation for the different approaches and clearly demonstrates the computational advantages of the Chandrasekhar scheme.

Another important consideration is that of stability of the schemes with respect to the increase in the time step. In Tables II(a) and II(b), as an indication of the stability of the various integrators, the averaged results of the experiment as in Case 2 above performed for a time step of $\Delta t=0.5$ are displayed in comparison with those with time step of $\Delta t=0.1$. The percentage errors in the average lengths and standard deviations are with respect to the corresponding values for $\Delta t=0.1$. It is again seen that the Chandrasekhar scheme outperforms the approximate schemes as the degradation of the results with increasing time step is comparatively small using this scheme.

A qualitative argument for determining the range of validity of the approximate schemes can be pursued as follows: As $T$ increases, the amplitudes of the random forces grow and the distribution function for the impulse becomes wider. Small disturbances are found either for a process taking place in an overall small temperature range or at the early times in the transient response of a system that is exposed to large temperatures. Hence, for $T$ small, the energy of the system is insufficient to allow barrier crossings, so that the system does not undergo large changes. Second, the momenta experienced by the system in different random realizations become comparable as they are selected from a narrow distribution. As a result, the system becomes quite deterministic and selecting many realizations within the time increment $\Delta t$ becomes unnecessary. At the extreme of very high temperatures, the barrier crossings become very frequent and the chain shortens to such an extreme that the differences between the various modes of computing again become negligible, in spite of the broadening of the momentum distribution function. In contrast, there is a comparatively large discrepancy between the predictions of the molecule length made by the approximate methods and the Chandrasekhar approach in the transient region and at the intermediate range for the temperature.

\section{CONCLUSION}

Two basic elements constitute the formulation presented in this paper: local harmonic modes and Chandrasekhar's formula for the probability distribution for a convolution involving a random function. First, by locally linearizing the governing Langevin equations and employing an orthogonal change of coordinates an explicit solution for the displacement and velocity vectors $\mathbf{x}$ and $\dot{\mathbf{x}}$ is constructed. Second, Chandresekhar's formula is employed in deriving the distribution function for the displacements and the velocities coming from the random forces. The local mode analysis is essential for the use of the Chandrasekhar's formula, since we need the formal solution as a convolution of the random forces and the local Green's function.
As a more traditional alternative, the equations can be integrated by various numerical algorithms such as a Runge-Kutta, Gear, or Verlet after generating random forces and thereby reducing the equations to a deterministic set during each infinitesimal time interval. However, these integrations yield the correct Gaussian distribution for the associated impulses over each time interval only in the limit as the number of realizations goes to infinity. One way out of this shortcoming is to rely on a qualitative ergodicity argument: Although the impulses in each time interval are not Gaussian, since the integration is carried out over many time steps, typically several thousand, the overall behavior will be Gaussian. One of the aims of the paper is to test this conjecture as well as to investigate how rapidly the predictions of the method involving several realizations of the random force in each time step converge to the limit which is obtained by Chandrasekhar's formula. Of course, in this approach, the system of equations is deterministic for each random realization and can be integrated directly using any algorithm. However, for the purpose of clarifying the effect of the finite number of random realizations in each time interval, the integration method is taken to be the same as with the proposed method. The use of the same integrator eliminates therefore possible numerical errors and discrepancies due to different discretization schemes and leaves only the errors due to the finiteness of the number of random realizations in each infinitesimal time interval. The convergence to the limit value for increasing the number of random realizations in each time step is found to be rather slow and even 20 realizations in each interval did not yield satisfactory results. The main observations are that the approximation may be acceptable for (i) short times, (ii) small temperatures, and (iii) average values over very long times. In particular, these approximations fail to give accurate results for transient phenomena, show slow convergence with increasing number of random realizations $M$ in each time interval and predict unreliable values for the standard deviation even in the steady regime. Moreover, the integration schemes such as Runge-Kutta, Gear, or Verlet are essentially local polynomial approximation of the particle coordinates. In contrast to these, the present scheme deals with trigonometric function representations through the local mode analysis. As a result, this permits much larger time steps for comparable accuracy. A subsequent article will investigate the comparison of the proposed method with the predictions of algorithms used for integrating the momentum equation that lead to an exponential kernel while again making use of Chandrasekhar's formula.

In conclusion, the proposed method, (i) incorporates the mathematically and conceptually correct limit for the probability distribution function for the displacements and velocities, (ii) is quite stable with respect to increases in the value of the time increment as well as in terms of fluctuations characterized by the variance, (iii) leads to considerable savings in computer time over the approximate method, and (iv) has the proper description during the transient regime, which is usually the most interesting phase of dynamical processes. 


\section{ACKNOWLEDGMENTS}

A.A. gratefully acknowledges the useful discussions he had with Professor J. H. Weiner of Brown University. R.O. is grateful to the Royal Society in cooperation with the Turkish Scientific Commission for a European Science Exchange Fellowship. The Bogaziçi University Research Fund is acknowledged for providing computing resources. H.R. acknowledges support from the U.S. Office of Naval Research.

\section{APPENDIX A: HARMONIC OSCILLATOR}

Consider the damped harmonic oscillator subjected to the random force $R(t)$

$$
\begin{aligned}
& \ddot{x}=-x-2 \eta \dot{x}+R(t), \\
& x(0)=x_{0} \quad \dot{x}(0)=\dot{x}_{0},
\end{aligned}
$$

where $\eta$ is a damping factor and $R=R(t)$ is the random external force acting on the system and satisfying the statistical relations (see Ref. 5)

$$
\begin{aligned}
& \langle R(t)\rangle=0, \\
& \left\langle R(t) R\left(t^{\prime}\right)\right\rangle=4 \eta T \delta\left(t-t^{\prime}\right),
\end{aligned}
$$

with $T$ being the temperature.

\section{A. Present algorithm}

The exact formal solution of Eq. (A1) is

$$
\begin{aligned}
x= & x_{0}+e^{-\eta t}\left\{x_{0} \cos \omega t+(1 / \omega)\left(\eta x_{0}+\dot{x}_{0}\right) \sin \omega t\right\} \\
& +(1 / \omega) \int_{0}^{t} e^{-\eta\left(t-t^{\prime}\right)} \sin \omega\left(t-t^{\prime}\right) R\left(t^{\prime}\right) d t^{\prime}, \\
\dot{x}= & e^{-\eta t}\left\{\dot{x}_{0} \cos \omega t-\left[\omega\left(1+K^{2}\right) x_{0}+K \dot{x}_{0}\right] \sin \omega t\right\} \\
& +\int_{0}^{t} e^{-\eta}\left(t-t^{\prime}\right)\left[\cos \omega\left(t-t^{\prime}\right)\right. \\
& \left.-K \sin \omega\left(t-t^{\prime}\right)\right] R\left(t^{\prime}\right) d t^{\prime},
\end{aligned}
$$

where

$$
\omega^{2}=1-\eta^{2} \quad K=\eta / \omega
$$

Following Chandrasekhar's formula, the convolutions above are Gaussian distributed with zero mean and respectively with the variances

$$
\begin{aligned}
\Sigma_{x}^{2}= & \frac{4 K T}{\omega} \int_{0}^{t} e^{-2 \eta\left(t-t^{\prime}\right)} \sin ^{2} \omega\left(t-t^{\prime}\right) d t^{\prime} \\
= & T\left\{1+(K / \omega) e^{-2 \eta t}[-(1 / \eta)+\eta \cos 2 \omega t\right. \\
& -\omega \sin 2 \omega t]\}, \\
\Sigma_{v}^{2}= & 4 \eta T \int_{0}^{t} e^{-2 \eta\left(t-t^{\prime}\right)}\left[\cos \omega\left(t-t^{\prime}\right)-K \sin \omega\left(t-t^{\prime}\right)\right]^{2} d t^{\prime} \\
= & T\left[1+(K / \omega) e^{-2 \eta t}(-(1 / \eta)\right. \\
& +\eta \cos 2 \omega t+\omega \sin 2 \omega t)] .
\end{aligned}
$$

It is seen that a closed form solution is obtained by this method.

\section{B. Ermak ${ }^{1,11-13}$ and Allen ${ }^{1,17,18}$ algorithms}

In these algorithms, the dynamical equation is rearranged as

$$
\begin{aligned}
& \dot{v}+2 \eta v=-x+R(t), \\
& \dot{x}=v .
\end{aligned}
$$

The algorithm for integrating this equation is achieved by the discretization below which propagates the solution at time $t$ to that at time $t+\Delta t$ as

$$
\begin{aligned}
& x(t+\Delta t)=x(t)+c_{1} \Delta t v(t)+c_{2} \Delta t^{2}[-x(t)]+\delta x^{G}, \\
& v(t+\Delta t)=c_{0} v(t)+c \Delta t[-x(t)]+c^{\prime} \Delta t[-x(t+\Delta t)]+\delta v^{G},
\end{aligned}
$$

where the numerical coefficients are

$$
\begin{aligned}
& c_{0}=e^{-2 \eta \Delta t} c_{1}=\left(1-c_{0}\right) / 2 \eta \Delta t, \quad c_{2}=\left(1-c_{1}\right) / 2 \eta \Delta t, \\
& c=c_{1}, \quad c^{\prime}=0 \quad(\text { Ermak algorithm) } \\
& c=c_{1}-c_{2}, \quad c^{\prime}=c_{2} \quad \text { (Allen algorithm) }
\end{aligned}
$$

and

$$
\begin{aligned}
& \delta x^{G}=(2 \eta)^{-1} \int_{0}^{t}\left[1-e^{-2 \eta\left(t+\Delta t-t^{\prime}\right)}\right] R\left(t^{\prime}\right) d t^{\prime}, \\
& \delta v^{G \underline{t}}=\int_{0}^{t} e^{-2 \eta\left(t+\Delta t-t^{\prime}\right)} R\left(t^{\prime}\right) d t^{\prime} .
\end{aligned}
$$

Again, $\delta x^{G}$ and $\delta v^{G}$ are selected from the Gaussian distributions with zero mean and the variances

$$
\begin{aligned}
\Sigma_{x}^{2} & =4 K T \int_{0}^{t}\left[1-e^{-2 \eta\left(t+\Delta t-t^{\prime}\right)}\right]^{2} d t^{\prime} \\
& =T(\Delta t / 2 \eta)\left[2-(2 \eta \Delta t)^{-1}\left(3-4 e^{-2 \eta \Delta t}+e^{-4 \eta \Delta t}\right)\right],
\end{aligned}
$$

$$
\Sigma_{v}^{2}=4 \eta T \int_{0}^{t} e^{-4 \eta\left(t+\Delta t-t^{\prime}\right)} d t^{\prime}=T\left(1-e^{-4 \eta \Delta t}\right) .
$$

Two significant differences should be noticed between the present algorithm and those of Ermak and Allen. First, in the present algorithm the correlation of the random force with $x$ is exact, incorporating the deterministic force $-x$, while in the latter this correlation is absent. This is clearly 

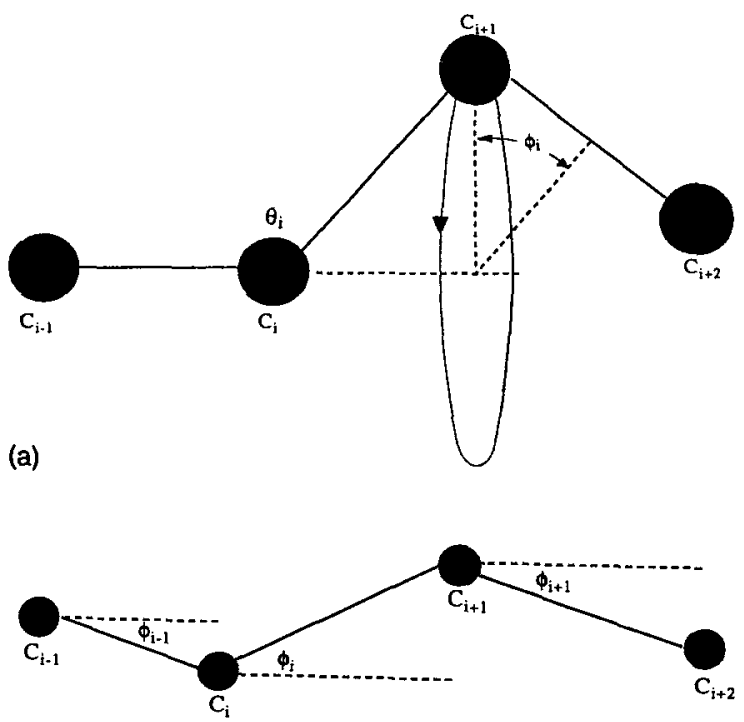

(b)

FIG. 3. (a) Section of three dimensional idealized chain molecule. (b) Two dimensional projection of the molecule.

demonstrated by the difference in the respective kernels of the two formulations. Second, the latter formulation is restricted to a limited value of $\Delta t$ by its nature, while the former has the closed form solution for all values of $t$. In practice, the restoring force of the oscillator will not be linear but a nonlinear function of $x$. In the proposed algorithm, the force will be linearized and the solution will be evolved in space to distances where the linearization is valid. This is the consideration that will determine the step size. In the latter algorithms on the other hand, the step size in time will be determined by the period of the oscillations. Certainly, the present algorithm will allow much larger time steps.

\section{APPENDIX B: THE POLYMER MODEL FOR THE CALCULATIONS}

The aim of the present work is the development of numerical techniques that may be employed in the simulation of complex polymer behavior under different conditions of stress and temperature. Following Weiner and Pear $^{3}$ we consider a three dimensional chain molecule, having $n$ atoms, a section of which is shown in Fig. 3(a). The valence angle $\theta_{j}$ between the interatomic bonds $C_{j-1} C_{j}$ and $C_{j} C_{j+1}$ remains fixed while rotation of $C_{j} C_{j+1}$ is allowed around an axis formed by $C_{j-1} C_{j}$. In addition, stretching of interatomic bonds cannot take place. The unknowns in the model are therefore the angles $\phi_{j}$ through which $C_{j} C_{j+1}$ has rotated relative to some equilibrium configuration, and the bond lengths between adjacent atoms.

A two dimensional projection of the model is shown in Fig. 3(b). The angles are measured relative to some fixed reference line which will be termed as the $x$ axis so that rotation is now visualized as taking place about axes perpendicular to the two dimensional plane. We now project the atoms onto the $x$ axis and assign the ordinate $x_{j}$ to the projection of the atom $C_{j}$ where

$$
x_{0}=0, \quad x_{j}=a \cos \phi_{j-1}+x_{j-1},
$$

and $a$ is the unstretched inter-atomic bond length. The potential $V$ is expressed as a function of the relative distances $\zeta_{j}=x_{j}-x_{j-1}(j=1,2, \ldots, N)$. The equilibrium positions a correspond to angles $\phi_{j}=0$ and $\pi$ whereas the rotational barrier at $\xi_{j}=0$ corresponds to angles $\phi_{j}=\pi / 2$ and $3 \pi / 2$. In the present paper we consider a double-well potential of the type defined by a quartic

$$
V(\zeta)=\gamma\left(1-\zeta^{2}\right)^{2}
$$

The original model of Weiner and $\mathrm{Pear}^{3}$ considered a piecewise quadratic potential that lends itself to a partial analytic solution.

\section{APPENDIX C: EVALUATIONS OF THE INTEGRALS OF RANDOM FUNCTIONS}

The following results are not new. However, the presence of the derivations makes the paper self-contained. In addition, the derivations are rephrased within the context of the paper. Lemma 1 concerns the integral of a random function of the type given in Eq. (2.12) while Lemma 2 is its generalization to the convolution of the random function with a deterministic function $\psi$. It should be noticed that Lemma 2 reduces to Lemma 1 for the special case of $\psi \equiv 1$.

\section{Lemma 1: If}

$$
R_{j}=2 \sqrt{\frac{\eta T}{\delta t}}(-1)^{\theta}
$$

where $\theta$ is an even or odd integer with equal probability over each subinterval of length $\delta t$, then as $\delta t / \Delta t \rightarrow 0$

$$
B_{j}(\Delta t)=\int_{0}^{\Delta t} R_{j}\left(t^{\prime}\right) d t^{\prime},
$$

has the Gaussian probability distribution with zero mean and variance $\sigma^{2}$ where

$$
\sigma^{2}=4 \eta T \Delta t .
$$

Proof: With the notation of Sec. III

$$
B_{j}(\Delta t)=\int_{0}^{\Delta t} R_{j}\left(t^{\prime}\right) d t^{\prime}=\sum_{k=1}^{M} 2 \sqrt{\eta T \delta t}(-1)^{\theta j k}
$$

where $\theta_{j k}$ is the $k$ th value of the random variable $\theta$ as realized in the $j$ th component $R_{j}$ of $\mathbf{R}$. By the central limit theorem ${ }^{26}$ as $\delta t / \Delta t \rightarrow 0 \quad B_{j}(\Delta t)$ has a Gaussian distribution with mean zero and standard deviation $\sigma_{j}$ given by

$$
\sigma=2 \sqrt{\eta T \delta t} \sqrt{M}=2 \sqrt{\eta T \delta t} \sqrt{\frac{\Delta t}{\delta t}}=2 \sqrt{\eta T \Delta t} .
$$

Lemma 2: If

$$
R_{j}=2 \sqrt{\frac{\eta T}{\delta t}}(-1)^{\theta},
$$


where $\theta$ is an even or odd integer with equal probability over each subinterval of length $\delta t$, then as $\delta t / \Delta t \rightarrow 0$

$$
I_{l}=\int_{0}^{\Delta t} \psi\left(\Delta t-t^{\prime}\right) R_{l}\left(t^{\prime}\right) d t^{\prime}
$$

has the Gaussian probability distribution with zero mean and variance $\Sigma^{2}$ given by

$$
\Sigma^{2}=4 \eta T \int_{0}^{\Delta t} \psi^{2}\left(\Delta t-t^{\prime}\right) d t^{\prime}
$$

Proof: Consider $I_{L M}=\Sigma_{j=1}^{M} \psi(\Delta t-j \delta t) \int_{(j-1) \delta t_{l}}^{j \delta t} R_{l}\left(t^{\prime}\right) d t^{\prime}$. From Lemma 1

$$
\int_{(j-1) \delta t}^{j \delta t} R_{l}\left(t^{\prime}\right) d t^{\prime}
$$

has a Gaussian distribution with mean zero and standard deviation $2 \sqrt{\eta T \delta t}$ so that $I_{l M}$ has Gaussian distribution with mean zero and variance $4 \eta T \Sigma_{j=1}^{M} \psi^{2}(\Delta t-j \delta t) \delta t$. As $M \rightarrow \infty \quad$ (i.e., $\delta t / \Delta t \rightarrow 0) \quad I_{l M} \rightarrow I_{1} \quad$ and $\quad \Sigma_{j=1}^{M} \psi^{2}(\Delta t$ $-j \delta t) \delta t \rightarrow \int_{0}^{\Delta t} \psi^{2}\left(\Delta t-t^{\prime}\right) d t^{\prime}$ as required.

${ }^{1}$ M. P. Allen and D. J. Tildesley, Computer Simulation of Liquids (Clarendon, Oxford, 1989).

${ }^{2}$ S. A. Adelman, Chemical Reaction Dynamics in Liquid Solution, Sec. IV, Adv. Chem. Phys. Vol. 13, edited by I. Prigogine and S. A. Rice (Wiley, New York, 1984).

3J. H. Weiner and M. R. Pear, Macromolecules 10, 317 (1977).

'J. H. Weiner and R. E. Forman, Phys. Rev. B 10, 315 (1974).
${ }^{5}$ M. R. Pear and J. H. Weiner, J. Chem. Phys. 71, 212 (1979).

${ }^{6}$ M. R. Pear and J. H. Weiner, J. Chem. Phys. 72, 3939 (1980).

${ }^{7}$ E. Helfand, J. Chem. Phys. 69, 1010 (1978); E. Helfand, Bell Sys. Tech. J. 58, 2289 (1979).

${ }^{8}$ M. Fixman, J. Chem. Phys. 69, 1527, 1538 (1978).

${ }^{9}$ E. Helfand, Z. R. Wasserman, and T. A. Weber, J. Chem. Phys. 70, 2016 (1979).

${ }^{10}$ N. Go and H. A. Schergara, Macromolecules 9, 535 (1976).

${ }^{11}$ D. L. Ermak, Brownian Dynamics and Their Applications to Dilute Solutions, Rapport d'activite scientifique du CECAM (1976).

${ }^{12}$ D. L. Ermak and H. Buckholtz, J. Comput. Phys. 35, 169 (1978).

${ }^{13}$ D. L. Ermak and J. A. McCammon, J. Chem. Phys. 69, 1352 (1978).

${ }^{14} \mathrm{R}$. Morf and E. Stoll, Numerical Methods for the Calculation of Molecular Dynamics, Int. Ser. for Num. Math., edited by J. Descloux and J. Marti, Vol. 37, Proc. of the Coll. on Numer. Analysis (Birkhauser, Basel, 1977).

${ }^{15}$ T. Schneider and E. Stoll, Phys. Rev. B 17, 1302 (1978).

${ }^{16}$ P. Turq, F. Lantelme, and H. L. Friedman, J. Chem. Phys. 66, 3039 (1977).

${ }^{17}$ S. A. Adelman, J. Chem. Phys. 71, 4471 (1979).

${ }^{18}$ M. P. Allen, Mol. Phys. 40, 1073 (1980).

${ }^{19}$ M. P. Allen, Mol. Phys. 47, 599 (1982).

${ }^{20}$ W. F. van Gunsteren and H. J. C. Berentsen, Mol. Phys. 45, 637 (1982).

${ }^{21}$ C. S. Peskin and T. Schlick, Comm. Pure Appl. Math. 42, 1001 (1989).

${ }^{22}$ T. Schlick and C. S. Peskin, Comm. Pure Appl. Math. 42, 1001 (1989).

${ }^{23}$ T. Schlick, S. Figueroa, and M. Mezei, J. Chem. Phys. 94, 2118 (1991).

${ }^{24}$ S. Chandrasekhar, Rev. Mod. Phys. 15, 1 (1943).

${ }^{25}$ W. H. Press, B. P. Flannery, S. A. Teukolsky, and W. T. Vetterling, Numerical Recipes. The Art of Scientific Computing (Cambridge University, Cambridge, 1986).

${ }^{26}$ R. V. Hogg and A. T. Craig, Introduction to Mathematical Statistics (Macmillan, New York, 1978). 